\title{
Instanton effects on CP-violating gluonic correlators
}

\author{
Shingo Mori ${ }^{1,2}$, Julien Frison ${ }^{2}$, Ryuichiro Kitano ${ }^{1,2}$, Hideo Matsufuru ${ }^{2}$, and Norikazu Yamada ${ }^{1,2}$ \\ ${ }^{1}$ Department of Particle and Nuclear Physics, The Graduate University for Advanced Studies (Sokendai), \\ Tsukuba 305-0801, Japan \\ ${ }^{2}$ High Energy Accelerator Research Organization (KEK), Tsukuba 305-0801, Japan
}

\begin{abstract}
In order to better understand the role played by instantons behind nonperturbative dynamics, we investigate the instanton contributions to the gluonic two point correlation functions in the SU(2) YM theory. Pseudoscalar-scalar gluonic correlation functions are calculated on the lattice at various temperatures and compared with the instanton calculus. We discuss how the instanton effects emerge or disappear with temperature and try to provide the interpretation behind it.
\end{abstract}

\section{Introduction}

$\theta$-vacuum of QCD is the sum of degenerate vacua labeled by the integer winding number which, through the instanton effect, have transition between each other. In each transition, the difference of winding numbers in between two states is the topological charge. This superposition allows a phase parameter $\theta$, which appears only in non-perturbative contributions. In the high temperature above the critical temperature $T_{c}$, the susceptibility in terms of $\theta$, so called the topological susceptibility $\chi_{t}$, is well described by the instanton calculus.

The instanton effect, for instance, is involved in the strong CP problem. In the path integral, the non-zero $\theta$ comes into the CP-violating term of the QCD Lagrangian, $\theta F \tilde{F}$. From the observation of the electric dipole moments of nucleon, the vacuum angle $\theta$ is bounded by extremely small value. The fact that there is no reason in the Standard Model to have $\theta \simeq 0$ in nature is a long standing puzzle, namely the strong CP problem. Two candidates as a solution for this problem are related to the instanton. One is the Peccei-Quinn (PQ) mechanism [1-3], which introduces the axion field $a(x)$ to make the QCD vacuum conserve $\mathrm{CP}$ symmetry. The smallness of $\theta$ parameter is explained as the coefficients of $F \tilde{F}$ term in SM $\bar{\theta}=\theta+a(x) / f_{a}$ feel the axion potential $\chi_{t} \cos \bar{\theta}$ stemming from by the instanton effects followed by $\theta=-\langle a\rangle / f_{a}$ with the PQ scale $f_{a}$ much higher than the EW scale. The other solution is the case of the bare $u$ quark mass $m_{u}$ being zero, then the $\theta$ parameter is unphysical. The case in which the additive mass shift of $O\left(m_{d} m_{s} / \Lambda_{\mathrm{QCD}}\right)$ to the $u$ quark mass comes from the 't Hooft vertex originated from instanton effects. The ambiguity of the non-perturbative $u$ quark mass still leaves room for the statement $m_{u}=0$. [4]

In spite of the importance of the role of the instanton, the understanding of the instanton contribution from analytic calculation within quantum field theory is quite difficult except for particular situations. The instanton picture is valid only at high temperature and may not apply to lower temperature, on a fortiori to zero temperature. Let us briefly introduce the situation of the instanton calculus based on quantum field theory. Due to the tunneling nature between degenerate classical vacua with distinct 
winding number, the instanton solution is the classical solution of the SU(N) Yang-Mills action with topological charge $Q$. This solution is known as the BPST instanton solution at zero temperature, and HS caloron solution for trivial holonomy and KvBLL caloron solution for non-trivial holonomy at finite temperature. At zero temperature, the instanton calculus of observables is ill-defined due to the IR divergence which essentially stems from the asymptotic freedom of QCD. While, at finite temperature, the temperature effect introduces cutoff for the long range interaction at scale $x \sim 1 / T$ due to the Debye screening, then all observables are IR finite. Especially, at very high temperature the interaction between instantons can be neglected and the dilute instanton gas approximation (DIGA) works well. The temperature dependence of the topological susceptibility $\chi_{t}$ calculated by DIGA is checked by the quenched lattice simulation. [5] However, at somewhat lower temperature, the interaction between instantons cannot be neglected and DIGA becomes invalid.

Our interests on the instanton are following three points. The first point is whether the instanton picture makes sense in in the local observables such as the correlator as well as a global observable such as the topological susceptibility. The second point is, in the finite temperature regime, from which temperature the instanton picture makes sense. Although the instanton picture of the topological susceptibility is established at high temperature in pure Yang-Mills theory, there is still ambiguity about the temperature where the dilute instanton gas approximation starts working. The third point is that if we consider the observable which has IR finite instanton contribution at zero temperature, such observable may comply with the instanton picture even at zero temperature.

The SU(2) Yang-Mills theory is interesting in the last context. The gluonic 2-point-correlation function $\left\langle\mathrm{T} F^{2}(x) F^{2}(0)\right\rangle$ is IR finite [6] even at zero temperature, due to the higher power of the instanton size suppression coming from the field strength in the instanton background. There may be still room for the instanton to play an important role at zero temperature, especially in this class of observables. However, we don't know how the instanton effects show up in the correlator calculated through the lattice simulation even at higher temperature where the instanton picture works in other global observables.

In this work we firstly investigate the case of finite temperature focusing on the CP-violating (CPV) gluonic correlator, where we can examine the first and the second point proposed above. In order to prove the validity of the instanton picture from the lattice simulation, it is easy to consider the observables which are dominated by the non-perturbative instanton contribution rather than the perturbative contribution in terms of the order counting of the strong coupling and the operator product expansion. This is why we choose the CPV gluonic correlator.

\section{Instanton calculus}

We will briefly introduce the outline of the calculation of the thermal instanton contribution to the CPV 2pt-gluon correlation function. Due to the self-duality of the instanton solution, $F F=F \tilde{F}$, three combinations of four dimensional operator $F F$ and $F \tilde{F}$ have same $x$-dependence. In the following, the CPV correlator will be denoted as $\langle s q(x)\rangle$ using the action density $s(x) \propto F F(x)$ and the topological charge density $q(x) \propto F \tilde{F}(x)$. At zero temperature, the leading instanton contribution to this function is derived by Dine et al. [6] as

$$
\left\langle\mathrm{T}^{2}(x) F^{2}(0)\right\rangle_{\theta}=c \Lambda^{b} x^{-b+8} \sin \theta,
$$

where subscript $\theta$ denotes the average in the $\theta$-vacuum and $b$ is the beta-function coefficient in SU(2) Yang-Mills theory, namely $b=22 / 3$.

At finite temperature we first calculated the leading instanton contribution. Since the temperature generates a cutoff for the interaction at distance $x \sim 1 / T$, the $x$-dependence of the correlator undergoes 
a non-trivial change because of the temperature effect. Naively, the leading instanton contribution to the correlator can be written as

$$
\langle s q(x)\rangle_{\theta}=c \Lambda^{b} x^{-\gamma(x)} T^{-b-\gamma(x)+8},
$$

where $\gamma(x)$ should be determined by the direct calculation.

The thermal instanton density in pure Yang-Mills theory with finite temperature is defined through the partition function of topological charge $Q=0$ and 1 sector in the semi-classical approximation. The quantum (=thermal) fluctuation come into the distribution $n(\rho, T)$ of the size $\rho$ and the position $z=\left(\vec{z}, \tau_{0}\right)$ of the instanton.

$$
\frac{Z_{Q=1}}{Z_{Q=0}}=\int_{0}^{\beta} d \tau_{0} \int d^{3} z \frac{d \rho}{\rho^{5}} n(\rho, T), n(\rho, T) \propto(\mu \rho)^{\beta} \exp \left[-\frac{2 N}{3}(\pi \rho T)^{2}\right],
$$

where $N$ is the number of colors and $\mu$ denotes the renormalization scale. We use a 1-loop level result for the instanton density, so $\beta=11 N / 3$. The full formulae are shown in our previous paper. [5] Note that the temperature fluctuation comes into the IR cutoff in terms of the instanton size $\rho$ with cutoff scale $\rho_{\text {cut }} \sim 1 / \pi T$.

The leading instanton contribution to the gluon 2-pt correlator is calculated using the background field method with instanton background. In the semi-classical approximation, the gluon correlator in the instanton background is written using the thermal instanton density as

$$
\left\langle\mathrm{T}^{2}(\vec{x}, \tau) F^{2}(\overrightarrow{0}, 0)\right\rangle_{I=1} \approx \int_{0}^{\beta} d \tau_{0} \int d^{3} z \frac{d \rho}{\rho^{5}} n(\rho, T) F_{\text {Inst. }}^{2}(\vec{x}, \tau) F_{\text {Inst. }}^{2}(\overrightarrow{0}, 0),
$$

where $F_{\text {Inst. }, \mu v}(x)$ is the field strength tensor constructed from the thermal instanton solution $A_{\mu}(x)$. Thus, the contribution from one instanton requires the integration of the product of field strength tensor with instanton density in terms of the instanton size $\rho$ and the instanton position $z$ in the whole region.

The thermal instanton solution with trivial holonomy is known as HS-caloron

$$
\begin{aligned}
A_{\mu}(x) & =i \tau_{\mu \nu}^{-} \partial_{\nu} \ln \Pi(x), \\
\Pi(x) & =1+\frac{2 \lambda^{2}}{R} \frac{\operatorname{sh} R}{\operatorname{ch} R-\cos R_{4}} \approx 1+\frac{4 \lambda^{2}}{\mathcal{R}^{2}}, \\
R & =2 \pi T|\vec{x}-\vec{z}|, R_{4}=2 \pi T\left(x_{4}-z_{4}\right), \mathcal{R}^{2}=R_{\mu} R_{\mu}, \lambda=\pi \rho T,
\end{aligned}
$$

where $\tau_{\mu \nu}^{ \pm}=\frac{1}{4}\left(\tau_{\mu}^{ \pm} \tau_{\nu}^{\mp}-\tau_{v}^{ \pm} \tau_{\mu}^{\mp}\right),\left\{\tau_{\mu}^{ \pm}\right\}=\left\{\tau^{a}, \pm i\right\} \quad(a=1,2,3)$ and $\tau^{a}$ is a Pauli matrices. This solution is in so-called singular gauge, which has a pole at the instanton position, namely $\mathcal{R}=0$. However, the calculation of the correlator should be done in the regular gauge. This singularity can be removed by a periodic singular transformation ${ }^{1}$,

$$
g=\frac{i \tau_{\mu}^{+} \bar{R}_{\mu}}{\sqrt{\bar{R}^{2}}}, \bar{R}_{\mu}=\left(R_{i}, \sin R_{4}\right)
$$

After the transformation, we obtain the thermal instanton potential in the regular gauge $A_{\mu}^{\prime}(x)=$ $g A_{\mu}(x) g^{-1}+g \partial_{\mu}^{x} g^{-1}$. The regularity of this function is checked as below. In the vicinity of the instanton position $z$, following approximation is valid,

$$
A_{\mu}(x) \approx f\left(\mathcal{R}^{2}\right) g^{-1} \partial_{\mu}^{x} g, g^{-1} \partial_{\mu}^{x} g \approx 2 \pi T \frac{-2 \tau_{\mu \nu}^{+} R_{v}}{\mathcal{R}^{2}}
$$

\footnotetext{
${ }^{1}$ This is not an gauge transformation. This transformation changes gauge invariant quantities such as $\operatorname{tr} F_{\mu \nu} F_{\mu \nu}$.
} 
Figure 1. The $x$-dependence of the leading instanton contribution to the gluon 2-pt correlator.

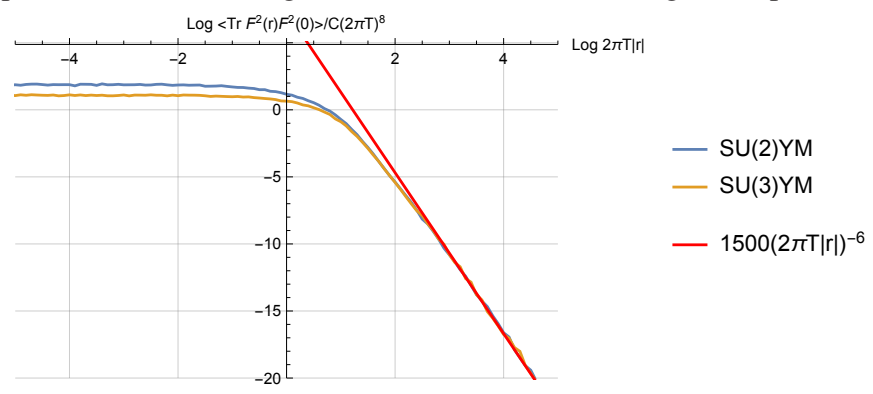

Table 1. Lattice parameters, physical temperatures, statistics

\begin{tabular}{llll}
\hline Lattice & $\beta$ & $T / T_{c}$ & $N_{\text {conf }}(|Q|=1)$ \\
\hline $24^{3} \times 6$ & 2.322 & 0.7215 & 122 \\
$24^{3} \times 6$ & 2.468 & 1.163 & 87 \\
$32^{3} \times 8$ & 2.582 & 1.246 & 154 \\
$32^{3} \times 8$ & 2.714 & 1.857 & 90 \\
\hline
\end{tabular}

where $f\left(\mathcal{R}^{2}\right) \equiv 4 \lambda^{2} /\left(4 \lambda^{2}+\mathcal{R}^{2}\right)$. Thus, the transformed gauge field behaves near the instanton position as

$$
A_{\mu}^{\prime}(x) \approx\left(1-f\left(\mathcal{R}^{2}\right)\right) g \partial_{\mu}^{x} g^{-1}=\frac{\mathcal{R}^{2}}{4 \lambda^{2}+\mathcal{R}^{2}} \times 2 \pi T \frac{-2 \tau_{\mu \nu}^{+} R_{v}}{\mathcal{R}^{2}}=-2 \pi T \frac{2 \tau_{\mu \nu}^{+} R_{v}}{4 \lambda^{2}+\mathcal{R}^{2}} .
$$

Substituting the regular instanton solution $A_{\mu}^{\prime}(x)$ into the integrand of Eq. (1), we calculated it numerically and eventually obtain figure. 1 . The horizontal axis denotes $\log (2 \pi T|\vec{x}|)$ and the vertical axis denotes $\log \left[\left\langle\mathrm{T} F^{2}(\vec{x}, \tau) F^{2}(\overrightarrow{0}, 0)\right\rangle_{I=1} / C(2 \pi T)^{8}\right]$, where $C$ is unimportant constant. The behavior of gluon correlator is $x^{-6}$ if $2 \pi T x \gg 1$ and constant if $2 \pi T x \ll 1$. The shape of curve is same in any temperature because the resulting formula is a function of not $x$ but $2 \pi T x$. If we choose the reference temperature as $T_{c}$, namely this plot is shifted by $\ln \left(T / T_{c}\right)$ in the horizontal direction. We will compare this plot with the CP-violating 2pt-gluon correlator calculated by lattice simulation, in which the region around $\log (2 \pi T|\vec{x}|) \sim 1$ will be compared in Sec. 5. In this comparison, we will use the horizontal axis as $\ln \left(2 \pi T_{c} x\right)$.

\section{Simulation setup}

The gauge configurations are generated on two lattices, using the Wilson gauge action with $N_{c}=2$ and Hybrid Monte Carlo algorithm with the Omelyan integrator. The lattice size is $24^{3} \times 6$ and $32^{3} \times 8$. The code is implemented by modifying the Bridge++ code set [7] so that SU(2) simulation can be performed. The physical temperatures, $T / T_{c}$, listed in table. 1 are derived by using non-perturbative beta function given by Engels et al. [8]. In order to reduce the residual statistical correlations of configurations, each configuration has separations of ten trajectories. The numbers of configuration listed in table. 1 count out the configurations which have topological charge $Q= \pm 1$, because they are used for the statistical analysis of the CPV gluonic correlator in the fixed topological charge sector. 
Figure 2. The result of the gradient flow with large flow time.

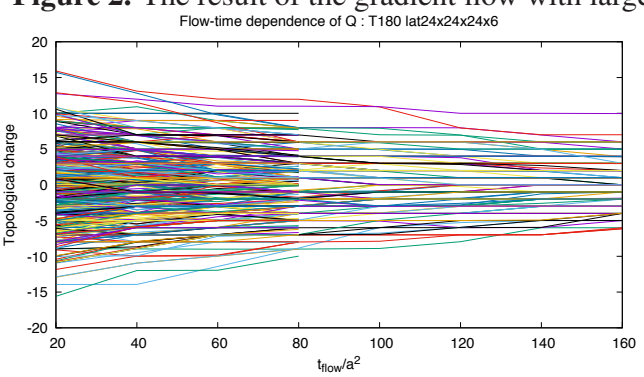

(a) $t_{\text {flow }}$ dependence of $Q$ in $24^{3} \times 6$ with $T / T_{c} \simeq 0.72$

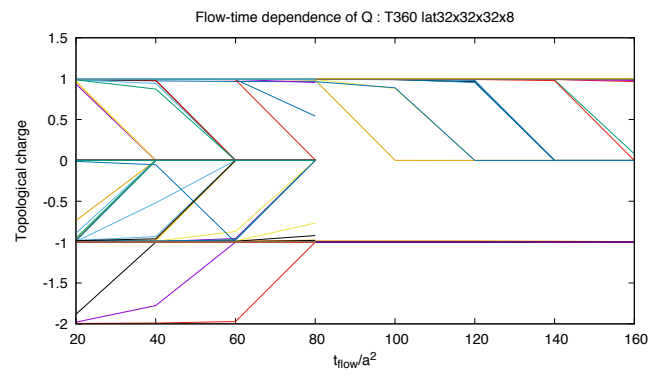

(c) $t_{\text {flow }}$ dependence of $Q$ in $32^{3} \times 8$ with $T / T_{c} \simeq 1.9$

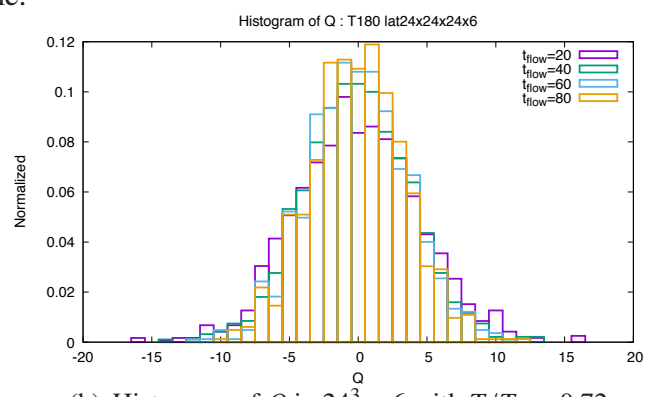

(b) Histogram of $Q$ in $24^{3} \times 6$ with $T / T_{c} \simeq 0.72$

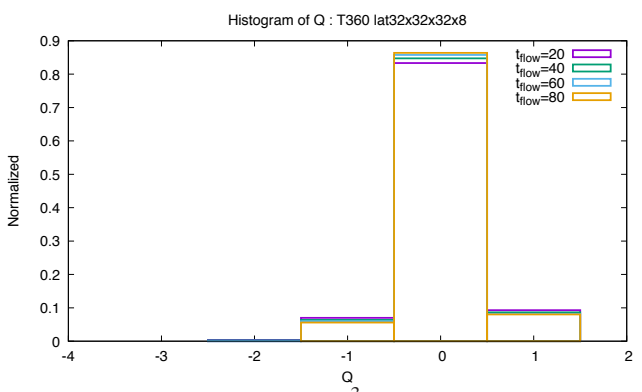

(d) Histogram of $Q$ in $32^{3} \times 8$ with $T / T_{c} \simeq 1.9$

\section{Gradient flow with large flow time}

The gradient flow in Yang-Mills theory $[9,10]$ is an evolution of gauge field in terms of the diffusion equation along the fictitious time $t$, so called flow time. In continuum theory the flow equation is

$$
\frac{\partial B_{\mu}(x, t)}{\partial t}=-\frac{\delta S_{\mathrm{YM}}}{\delta B_{\mu}(x, t)}, B_{\mu}(x, t=0)=A_{\mu}(x),
$$

where $A_{\mu}(x)$ is the gauge field in $4 \mathrm{~d}$ Euclidean Yang-Mills theory and $S_{\mathrm{YM}}$ is the Yang-Mills action. This procedure makes the field configuration smoothed with smearing radius $x \sim \sqrt{8 t}$, which corresponds to the diffusion length. Interestingly, the flow equation above does not modify the classical configuration especially the instanton, because the force becomes zero for stationary solutions. Then, the large flow time configuration approaches some classical solutions. In the lattice simulation at very high temperature, if the configurations generated via HMC algorithm have non-trivial topological charge, the configurations are considered as classical instanton configuration with quantum fluctuations. The flow extracts the classical instanton configuration without changing the instanton size and position. Thus even though the quantum fluctuation around the instanton solution is removed by the large flow, the instanton size distribution of flowed configurations still preserves the information of the instanton density $n(\rho, T)$ of the original gauge configurations. Although this reasoning only applies for very high temperature, we extrapolate this method for lower temperature in order to investigate from which temperature the instanton picture makes sense.

Figure. 2 shows the transition of the topological charge during the Wilson flow in the region $t / a^{2} \in[20,160]$ in the left column, and the distribution of the topological charge in four flow times, $t / a^{2}=20,40,60,80$ in the right column. The upper row shows results of lower temperature $T / T_{c} \simeq 0.72$ and the lower row shows ones of upper temperature $T / T_{c} \simeq 1.9$. Even when the flow time is above $t / a^{2}=80$, some configuration are not stable in terms of the topological charge. This 
Figure 3. The CP-violating gluonic correlator at fixed topological charge sector with $|Q|=1$.

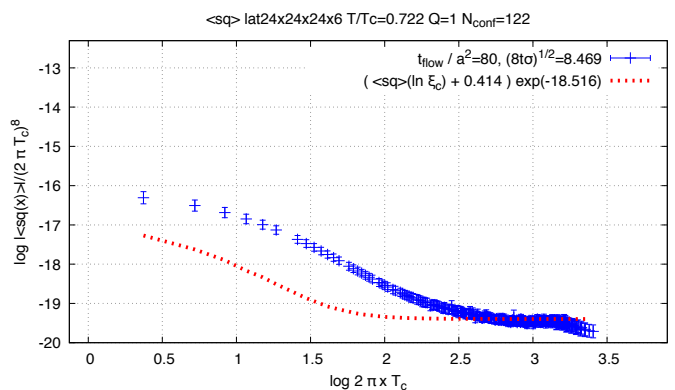

(a) CPV 2pt-function in $24^{3} \times 6$ with $T / T_{c} \simeq 0.72$

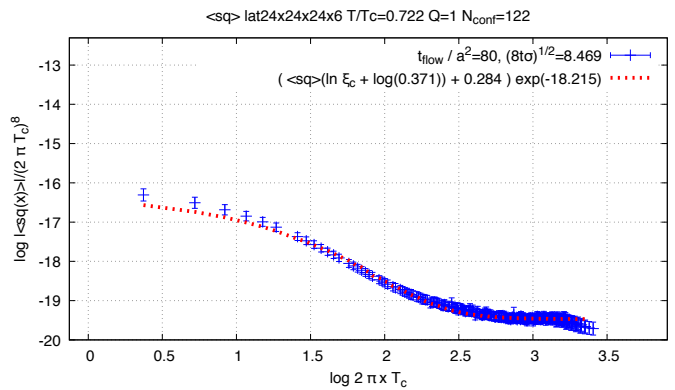

(c) CPV 2pt-function in $24^{3} \times 6$ with $T / T_{c} \simeq 0.72$

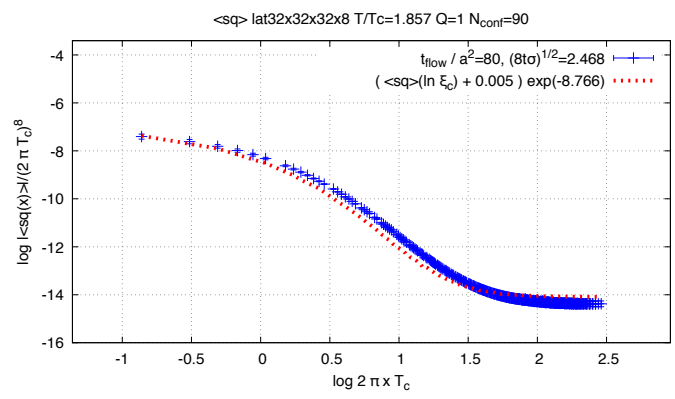

(b) CPV 2pt-function in $32^{3} \times 8$ with $T / T_{c} \simeq 1.9$

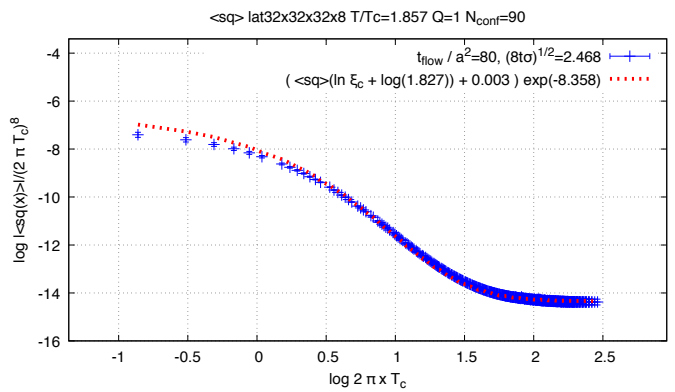

(d) $\mathrm{CPV}$ 2pt-function in $32^{3} \times 8$ with $T / T_{c} \simeq 1.9$

would occur due to the discretization error of the Wilson gauge action used in HMC and the Wilson flow. In the simulation of 2pt-gluon correlator shown in Sec. 5, we used the flowed configuration with $t / a^{2}=80$ to measure the topological charge and the CP-violating gluon correlator. The number of configuration with $|Q|=1$ listed in table. 1 is determined by the topological charge at $t / a^{2}=80$ as well.

\section{CP-violating gluonic correlator at fixed topological charge sector}

Using the configuration generated as described in Sec. 3 and evolved by the Wilson flow with large flow time described in Sec. 4, the result of the lattice simulation is compared with the instanton calculus described in Sec. 2.

In order to pay attention to the fact that the temperature effect introduces suppression for the long range interaction longer than scale $x \sim 1 / T$, we choose the horizontal axis as dimension-less $\xi_{c} \equiv \ln \left(2 \pi T_{c} x\right)$. In this case the horizontal position where cutoff appears differs by temperature with horizontal shift $\ln \left(T / T_{c}\right)$. As a result of numerical integration we have the CPV correlator in one instanton background as shown in figure. 1 as a function of $\xi_{c}$,

$$
\Pi_{I=1}^{\text {Inst. }}\left(\xi_{c}\right)=\frac{\left\langle s q\left(\xi_{c}\right)\right\rangle_{I=1}^{\text {Inst. }}}{C_{\text {Inst. }}\left(2 \pi T_{c}\right)^{8}},
$$

where $C_{\text {Inst. }}$ is unimportant constant and subscript $I=1$ denotes the one instanton background. In this function the horizontal axis is shifted from the plot shown in figure. 1 by the relation, $\ln \left(2 \pi T_{c} x\right)=$ $\ln (2 \pi T x)-\ln \left(T / T_{c}\right)$. 
On the other hand, the CPV correlator calculated via the lattice simulation is denoted as

$$
\Pi_{|Q|=1}^{\text {Lat. }}\left(\xi_{c}\right)=\frac{\left\langle s q\left(\xi_{c}\right)\right\rangle_{|Q|=1}^{\text {Lat. }}}{\left(2 \pi T_{c}\right)^{8}},
$$

where the ensemble average of the correlator is calculated using configurations with non-trivial topological charge $|Q|=1$. In this average, the sign of the correlator of configurations with $Q=-1$ is flipped. Here, the ensemble average of CPV correlator is zero when using all configuration generated by HMC algorithm or with $Q=0$.

Note that the result of the fixed topological charge sector $|Q|=1$ which is calculated via the lattice simulation is not exactly equal to one of the $I=1$. The unit topological charge denotes the sum of all sectors which has $n_{+}$instanton and $n_{-}$instanton satisfying $n_{+}-n_{-}=1$. In the finite volume simulation, it is conceivable that configurations with $Q=1$ generated by HMC algorithm contain up to several number of instanton or anti-instanton due to the limited box size. Below we assume that most of the configurations with $|Q|=1$ contain only one (anti-)instanton in the box and we can approximate the observables given by the lattice simulation as $\langle O\rangle_{|Q|=1} \approx\langle O\rangle_{I=1}$. We will compare the observables analytically calculated in the one instanton background, $\langle O\rangle_{I=1}$, and one with $|Q|=1$ numerically calculated using the lattice simulation, $\langle O\rangle_{|Q|=1}$.

Then we compared them in two ways. The first one adopts two parameters $A$ and $B$ for the overall factor and additional constant term, respectively. In our analysis, we are not interested in the overall factor of the instanton density which depends on the renormalization scale. The constant term $B$ denotes the contribution from disconnected part of the 2pt-function and the finite volume effect [11],

$$
A\left(\Pi_{|Q|=1}^{\text {Inst. }}\left(\xi_{c}\right)+B\right) \text { v.s. } \Pi_{|Q|=1}^{\text {Lat. }}\left(\xi_{c}\right)
$$

The second fitting introduces three parameters, the overall factor $A$, the constant term $B$ and the effective temperature $r=T^{\prime} / T_{c}$. In addition to the degree of freedom of the first fitting, we adopt the effective temperature $T^{\prime}$ to somehow understand the correlator calculated by the lattice simulation in which the cutoff temperature is different from what instanton calculus predicts.

$$
A\left(\Pi_{|Q|=1}^{\text {Inst. }}\left(\xi_{c} \times r\right)+B\right) \text { v.s. } \Pi_{|Q|=1}^{\text {Lat. }}\left(\xi_{c}\right) \text {. }
$$

Figure. 3 denotes the $\mathrm{CP}$-violating gluonic 2 pt-function calculated using the lattice simulation in blue line with $1 \sigma$ error and instanton calculus in red dotted line. In figure. 3 (a) and (b) the lattice result of $T / T_{c} \simeq 0.72$ and $T / T_{c}=1.857$ are fitted using Eq. (1), respectively. In the high temperature range, the correlator is well fit by these two parameters, so the instanton picture is valid in the high temperature regime as expected. However, in the low temperature the fit is not good. The correlator given by the lattice simulation shows larger correlation in the large distance than the instanton prediction. The position where power of $x$ is falling down represents the typical instanton size in the ensembles. The instanton profile produced by the lattice simulation behaves like one predicted by the instanton calculus at lower temperature than the input temperature. In figure. 3 (c) and (d), the lattice result of $T / T_{c} \simeq 0.72$ and $T / T_{c}=1.857$ respectively are fitted using Eq. (2). We obtain $r \simeq 0.37 \pm 0.021$ and $1.827 \pm 0.023$ for the lattice simulation with $T / T_{c} \simeq 0.72$ and 1.9 , respectively, where the statistical errors are determined by the jack-knife method. In the low temperature $T / T_{c}=0.72$, the instanton size distribution calculated by the lattice simulation behaves as that with effective temperature $T / T_{c} \simeq 0.37$.

\section{Conclusion and outlook}

The instanton effect is involved in the solution of the strong CP problem in the Standard Model. Existing knowledge about the instanton picture contains the ambiguities both at zero and finite temperatures. We try to clarify the role of the instanton picture behind the non-perturbative dynamics 
via the lattice simulation focusing on the CPV gluon 2 pt-function. Since, in very high temperature, the instanton size distribution of the configurations generated by HMC is unchanged under the gradient flow, the configurations after applying large flow still have information of the instanton size distribution behind the original configurations. We measure the typical size or cutoff determined by temperature $\sim 1 / T$ comparing the correlator of analytic result with one of numerical result. In the high temperature region, we found instanton picture describes the CPV correlator calculated by lattice simulation. In the low temperature region, we found the large size instantons have larger contribution than expected, which is equivalent to the case when instantons distribute with lower effective temperature. As a future work, we will investigate from which temperature the DIGA prediction is valid and how the CPV correlator behaves in the low temperature region with more points. We will also carry out the simulation with the Symantzik improved action in the HMC and gradient flow, which reduces the discretization error included in our current results.

\section{Acknowledgements}

This work is in part based on Bridge++ code (http://bridge.kek.jp/Lattice-code/). This work is supported by JSPS KAKENHI Grant No. 15H03669, 15KK0176, MEXT KAK- ENHI Grant No. 25105011 (RK), the Large Scale Simulation Program No. 16/17-28 of High Energy Accelerator Research Organization (KEK), and Interdisciplinary Computational Science Program No. 17a15 in CCS, University of Tsukuba.

\section{References}

[1] R. D. Peccei and H. R. Quinn, Phys. Rev. Lett. 38, 1440 (1977). doi:10.1103/PhysRevLett.38.1440

[2] R. D. Peccei and H. R. Quinn, Phys. Rev. D 16, 1791 (1977). doi:10.1103/PhysRevD.16.1791

[3] R. Kitano and N. Yamada, JHEP 1510, 136 (2015) doi:10.1007/JHEP10(2015)136 [arXiv:1506.00370 [hep-ph]].

[4] J. Frison, R. Kitano and N. Yamada, PoS LATTICE 2016, 323 (2016) [arXiv:1611.07150 [heplat]].

[5] J. Frison, R. Kitano, H. Matsufuru, S. Mori and N. Yamada, JHEP 1609, 021 (2016) doi:10.1007/JHEP09(2016)021 [arXiv:1606.07175 [hep-lat]].

[6] M. Dine, P. Draper and G. Festuccia, Phys. Rev. D 92, no. 5, 054004 (2015) doi:10.1103/PhysRevD.92.054004 [arXiv:1410.8505 [hep-ph]].

[7] Bridge++ project website, http://bridge.kek.jp/Lattice-code/.

[8] J. Engels, F. Karsch and K. Redlich, Nucl. Phys. B 435, 295 (1995) doi:10.1016/05503213(94)00491-V [hep-lat/9408009].

[9] M. Lüscher, JHEP 1008, 071 (2010) Erratum: [JHEP 1403, 092 (2014)] doi:10.1007/JHEP08(2010)071, 10.1007/JHEP03(2014)092 [arXiv:1006.4518 [hep-lat]].

[10] M. Luscher and P. Weisz, JHEP 1102, 051 (2011) doi:10.1007/JHEP02(2011)051 [arXiv:1101.0963 [hep-th]].

[11] S. Aoki, H. Fukaya, S. Hashimoto and T. Onogi, Phys. Rev. D 76, 054508 (2007) doi:10.1103/PhysRevD.76.054508 [arXiv:0707.0396 [hep-lat]]. 